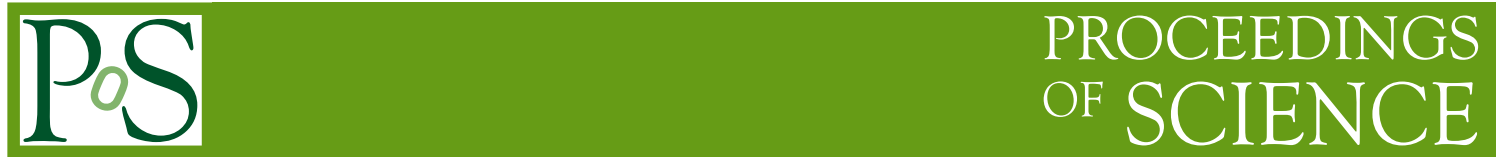

\title{
Flavor Physics at ATLAS and CMS
}

\author{
Nuno T. Leonardo*i \\ Purdue University \\ E-mail: nuno.leonardo@cern.ch
}

\begin{abstract}
Heavy quarks are copiously produced at the LHC offering new opportunities to improve our understanding of flavor physics. The ATLAS and CMS experiments have prepared to explore them through a broad program spanning from quarkonia studies, to precision measurements of $b$ hadron properties, to the search for rare decays and processes sensitive to new physics. Various initial measurements are invaluable, in addition, for the physics commissioning of the detectors with the earliest LHC collision data.
\end{abstract}

XXth Hadron Collider Physics Symposium

November 16 - 20, 2009

Evian, France

\footnotetext{
* Speaker.

${ }^{\dagger}$ On behalf of the ATLAS and CMS collaborations.
} 


\section{Introduction}

A new energy exploration domain is imminent with the start-up of the Large Hadron Collider (LHC) at CERN. Along come new opportunities to improve our understanding of flavor physics. Production properties of beauty and charm hadrons will be measured against the framework of quantum chromodynamics (QCD). The new data will allow measurements of $\mathrm{CP}$ violation and flavor changing processes with significant accuracy, and very rare decays will be searched for. A thorough program of precision measurements in the flavor sector will have the potential to detect effects of new physics beyond the standard model (SM) that may be awaiting to be revealed at the LHC, and allow to constrain the associated theory parameter space.

ATLAS [1] and CMS [2] are general purpose particle experiments operating at the LHC. The two detectors have been designed to explore the TeV energy frontier. Their characteristics, including excellent tracking and muon systems, in particular, with large pseudorapidity coverage, render them well suited for heavy flavor studies. An important part of the heavy flavor physics program will be carried out in the earlier stages of data taking. Many analyses dedicated to heavy flavor production are indeed viable already with $\mathscr{O}(10) \mathrm{pb}^{-1}$ integrated luminosity. Those studies to be performed with the very first data will constitute an important component of the physics commissioning of the detectors and triggers with collisions. ${ }^{1}$

\section{Heavy flavor production at the LHC}

Charm and beauty quarks are produced profusely at the LHC. Heavy quarkonium production has been studied extensively in various theoretical descriptions. The framework of non-relativistic QCD (NRQCD) through the introduction of the so-called color-octet mechanism (COM) had successfully accommodated the quarkonium differential cross section data from the Tevatron, owing in part to its adjustable parameters (long distance matrix elements). However, the polarization predictions based on COM are in strong contrast with observations [3, 4]. Other approaches, such as the color-singlet model (CSM), have been more recently revived. Quarkonia studies with early LHC data are expected to help resolving the current puzzling situation.

Three mechanisms contribute to beauty production at hadron colliders. The lowest-order twoto-two $b \bar{b}$ diagrams, involving gluon-gluon fusion and $q \bar{q}$ annihilation, are referred to as flavor creation (FC) in the hard scattering. Flavor excitation (FE) corresponds to diagrams where a $b \bar{b}$ pair from the quark sea of the proton is excited into the final state as one of the $b$ quarks undergoes a hard QCD interaction with a parton from the other proton. Gluon splitting (GS) refers to the soft processes where the $b \bar{b}$ pair arises from the splitting $g \rightarrow b \bar{b}$ in the initial or final state. The $b \bar{b}$ being the largest physics background for many processes of interest at the LHC, both within the standard model and beyond, it will be crucial to verify the ability for next-to-leading order perturbative QCD to accurately predict background characteristics and rates in the LHC data.

\section{Trigger}

Online event selection and rate reduction to a level appropriate for storage, $\mathscr{O}(200) \mathrm{Hz}$, is

\footnotetext{
${ }^{1}$ The first LHC collisions took place and were successfully registered by the experiments in December 2009.
} 


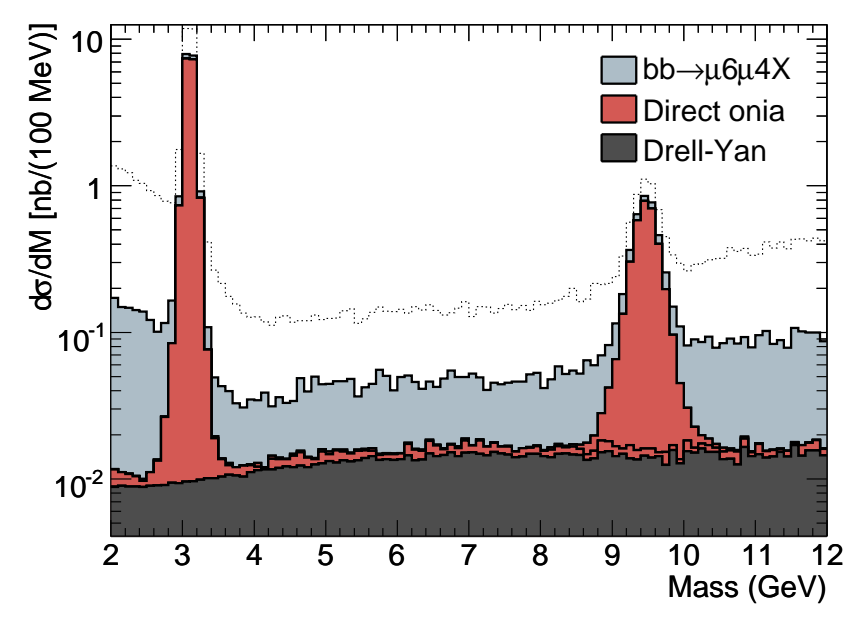

Figure 1: Invariant mass of dimuons from various sources, reconstructed with a $\mu 6 \mu 4$ trigger, with the requirement that both muons are identified as coming from the primary vertex and with a pseudo-proper time cut of $0.2 \mathrm{ps}$. The dotted line shows the cumulative distribution without vertex and pseudo-proper time cuts. Peaks from the $J / \psi$ and $\Upsilon(1 S)$ clearly dominate the background; no higher mass $\psi$ and $\Upsilon$ states are included. (ATLAS simulation)

achieved with a 2(3)-tiered trigger system at CMS (ATLAS) [1, 2]. The hardware trigger level (L1) is based on coarse information from the calorimeter and muon systems alone. In the ensuing, software-based trigger filtering (HLT) progressively more information from all detector systems becomes accessible to algorithms providing accuracies approaching those of offline reconstruction.

Heavy quark physics at CMS and ATLAS relies primarily on single and double-muon triggers. Different transverse momentum $p_{T}$ thresholds are anticipated, with the lowest threshold paths being prescaled triggers. Most of the CMS studies presented herein require at least one muon with $p_{T}>$ $3 \mathrm{GeV} / \mathrm{c}$, or two muons with $p_{T}>3 \mathrm{GeV} / \mathrm{c}$ (the $2 \mu 3$ path). For ATLAS, the required $p_{T}$ thresholds for the first and second muons are 6 and $4 \mathrm{GeV} / c(\mu 6 \mu 4)$, while at startup the threshold on the first muon may be lowered to $4 \mathrm{GeV} / c(2 \mu 4)$. Additional dedicated $B$-physics trigger signatures, with requirements on dimuon invariant mass and displaced vertices, have also been devised.

\section{Onia}

Quarkonia studies play an important role in early data taking. With an integrated luminosity of $10 \mathrm{pb}^{-1}$ ATLAS and CMS will be able to register samples of the order of $10^{5}$ events containing $J / \psi \rightarrow \mu^{+} \mu^{-}$selected by the low luminosity trigger menus. Quarkonia reconstruction will be employed for trigger and detector system calibration. A related procedure, based on the so-called tag-and-probe method, has been implemented to extract the low- $p_{T}$ muon trigger and reconstruction efficiencies using data.

\subsection{Cross section}

The measurement of the $J / \psi$ and $\Upsilon$ resonances production cross sections are expected to be amongst the first results extracted from the early data. Figure 1 illustrates the quarkonia signal and main background invariant mass distributions [5] in the dimuon mass range $2-12 \mathrm{GeV} / \mathrm{c}^{2}$, with reconstruction efficiencies and background suppression selection accounted for.

The precision tracking of ATLAS and CMS permits to disentangle the prompt $J / \psi$ production from $b$-hadron decays, thus also allowing the determination of the $b$-hadron cross section. 


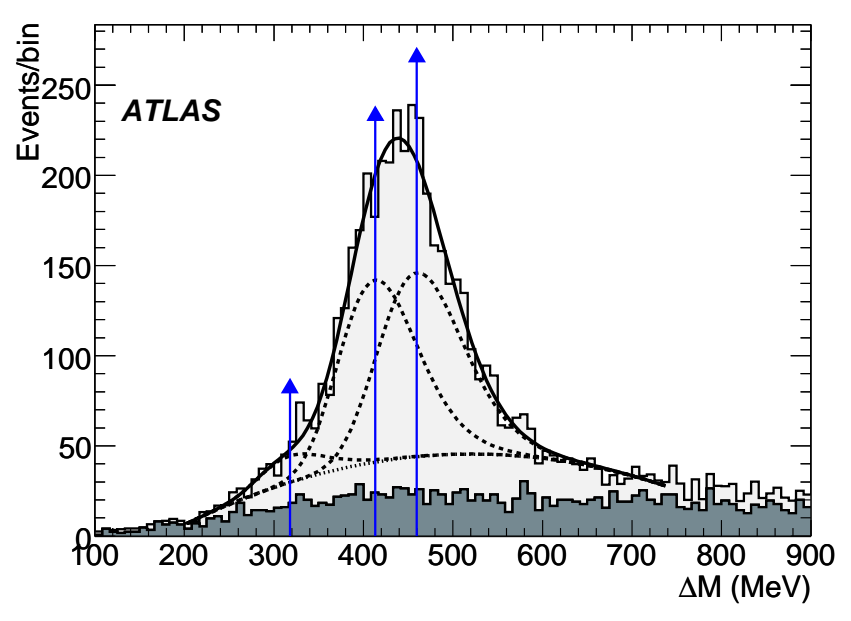

Figure 2: Difference in invariant masses of $\mu \mu \gamma$ and $\mu \mu$ systems in prompt $J / \psi$ events (light grey) with $b \bar{b}$ background surviving selection cuts (dark grey). The solid line is the result of a simultaneous fit to the measured distribution, with the position of the $\chi_{c 0}, \chi_{c 1}$ and $\chi_{c 2}$ signal peaks (indicated by arrows) fixed at their common values, and with a common resolution. (ATLAS simulation)

Contributions from prompt and non-prompt $J / \psi$ are statistically separated through a simultaneous fit to the observed $J / \psi$ candidates invariant mass and pseudo-proper decay time. Already with a small integrated luminosity of the order of $3 \mathrm{pb}^{-1} \mathrm{CMS}$ estimates that the precision of the result is limited by systematic uncertainties, at the $15 \%$ level [6]. The dominant systematic sources arise from the luminosity measurement and the dependence of the detector acceptance on the unknown production polarization.

A large fraction $\mathscr{O}(30-50 \%)$ of $J / \psi$ and $\Upsilon$ resonance production originates from the decay of heavier prompt states, such as $\chi_{c} \rightarrow J / \psi \gamma, \chi_{b} \rightarrow \Upsilon \gamma$. The reconstruction of the cascade $\chi_{c(b)}$ decays will be attempted by associating a reconstructed $J / \psi(\Upsilon)$ with the radiated photon. The distribution of the difference $\Delta M$ between the invariant masses of the $\mu \mu \gamma$ and $\mu \mu$ systems, obtained for selected $\chi_{c}$ candidates in a study performed by ATLAS [5], is shown in Figure 2. Event yields correspond to an integrated luminosity of $10 \mathrm{pb}^{-1}$. A similar analysis procedure can be applied to reconstruct the $\chi_{b}$ decay. However the higher dimuon mass, and hence smaller expected boost, makes the photon considerably softer and thus more difficult to detect. It is estimated that integrated sample sizes of up to $1 \mathrm{fb}^{-1}$ may be required for reliably observing $\chi_{b} \rightarrow \Upsilon \gamma$ decays.

\subsection{Polarization}

The measurement of the $J / \psi$ and $\Upsilon$ polarization will be important for discriminating and establishing the quarkonium hadro-production mechanisms. Quarkonium polarization is measured by analyzing the angular distribution of the muons produced in the decay. The polarization parameter $\alpha$ describes the distribution $d N / d \cos \theta \sim 1+\alpha \cos ^{2} \theta$. The angle $\theta$, measured in the $J / \psi(\Upsilon)$ center of mass, helicity frame, is the angle between the $\mu^{+}$momentum and the direction of motion of the quarkonium in the laboratory rest frame.

ATLAS plans to use both double $(\mu 6 \mu 4)$ and single $(\mu 10)$ muon triggers, for improved $|\cos \theta|$ coverage, for the $J / \psi$ analysis. In the case of the $\Upsilon, \mu 10$ should have too high background levels [5]. With an integrated luminosity of $10 \mathrm{pb}^{-1}$ it should be possible to measure the polarization of the $J / \psi$ with a precision of order $0.02-0.06$ in a wide range of transverse momenta. In the case of the $\Upsilon$ the expected precision for $\alpha$ is of order 0.20 . 


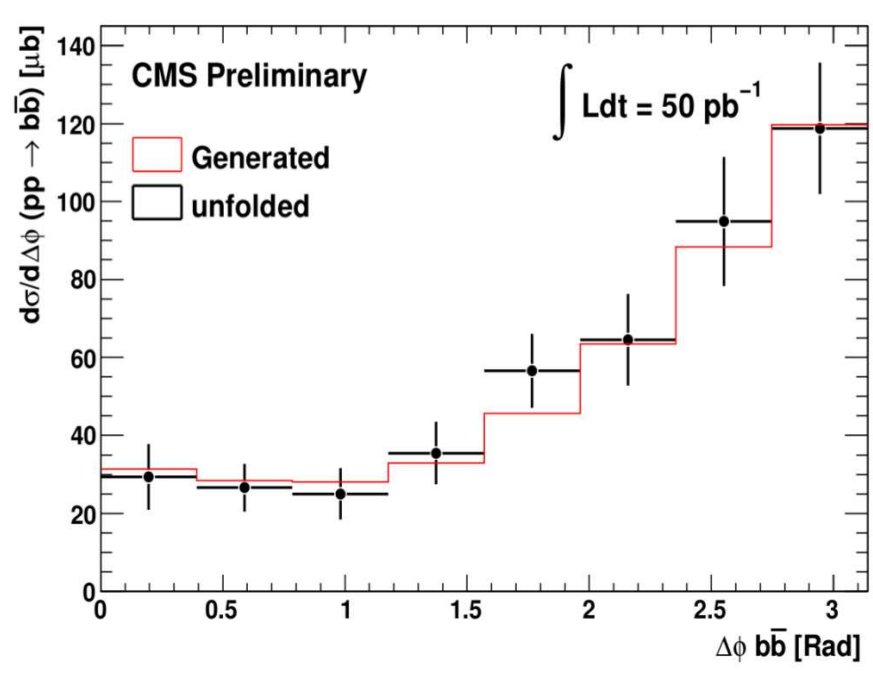

Figure 3: Differential $b \bar{b}$ production cross section $d \sigma / d \Delta \phi$ as a function of the $b$ quarks angular separation. The data points are obtained from a fit to the invariant $J / \psi$ mass, the transverse $J / \psi$ decay length and the distance of closest approach of the third muon to the beamline. The unfolding procedure is applied to correct the reconstructed $\Delta \phi$ distribution back to that of the original $b$ quarks. (CMS simulation)

\section{Inclusive studies}

\section{$5.1 b \bar{b}$ correlations}

Correlation measurements are foreseen at the LHC for studying details of the production mechanisms previously discussed: flavor creation, flavor excitation, gluon splitting. These can be statistically disentangled by assessing the angular separation $\Delta \phi$ between the two $b$ quark directions in the transverse plane. CMS plans to explore a clean, fully muonic signature with the first $50 \mathrm{pb}^{-1}$ of collision data [7]. The decay of one $b$ is tagged by reconstructing the $J / \psi \rightarrow \mu \mu$ decay. Events are also required to contain a second muon consistent with the semileptonic decay of the second $b$. Figure 3 shows the expected differential cross section $d \sigma / d \Delta \phi$ measurement. Depending on the particular $\Delta \phi$ bin, an accuracy of $15-25 \%$ can be obtained, combining statistical and systematic uncertainties. An accuracy at the $10 \%$ level is expected for the integrated total cross section.

\section{$5.2 b$ production}

The measurement of the inclusive $b$ quark production cross section is proposed by CMS for a high statistics sample $\mathscr{O}(10) \mathrm{fb}^{-1}$ of events containing jets and at least one muon [8]. The sample is collected with a single muon trigger at L1 and a 'muon $+b$-jet' at HLT. Event selection requires a $b$-tagged jet, where the tagging method is based on inclusive secondary vertex reconstruction and is characterized by a tagging efficiency of about $60 \%$. Each reconstructed muon is associated with the most energetic $b$-tagged jet, and must be closest to this jet than to any other jet. The transverse momentum of the muon with respect to the $b$-jet axis effectively discriminates between $b$ events and background, thereby allowing to extract the signal fraction from a template fit to the data.

The $b$ purity of the selected events varies as function of the transverse momentum in a range from $70 \%$ to $55 \%$. The largest systematic uncertainty on the cross section arises from a $3 \%$ uncertainty on the jet energy scale which leads to a cross section uncertainty of $12 \%$ for $E_{T}>50 \mathrm{GeV}$. Figure 4 shows the estimated uncertainty as function of the $b$-tagged jet transverse momentum. 


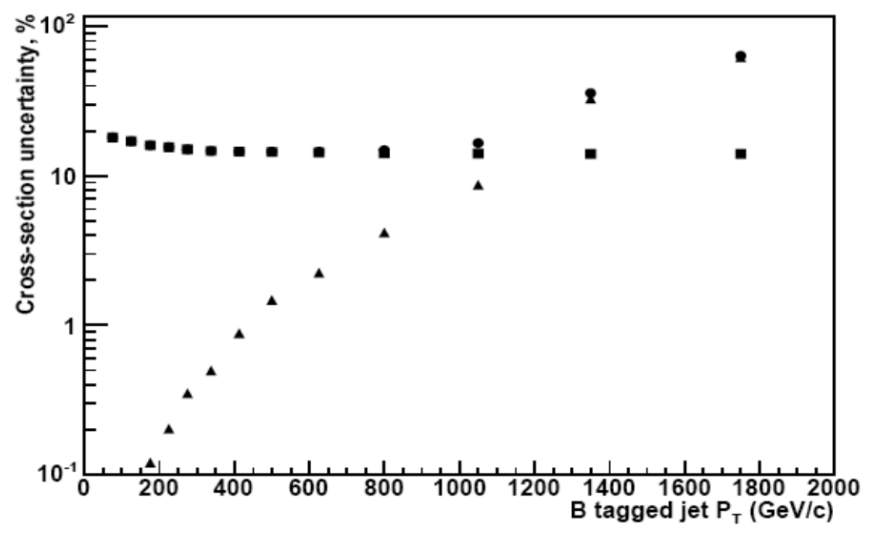

Figure 4: Expected uncertainty on the inclusive $b$ cross section measurement: statistical (triangles), systematic (squares), total (dots); shown as a function of the $b$-tagged jet transverse momentum with respect to the beamline. It may be inferred from the estimate a 1.5 $\mathrm{TeV} / c$ reach for the highest accessible $p_{T}$ of $B$ hadrons. (CMS simulation)

\section{Exclusive and rare decays}

\section{1 $B_{u} \rightarrow J / \psi K^{+}, B_{d} \rightarrow J / \psi K^{* 0}, B_{s} \rightarrow J / \psi \phi$}

The exclusive $B \rightarrow J / \psi K^{(*)}$ and $B_{s} \rightarrow J / \psi \phi$ decays will be observed with the first tens of inverse-picobarn of integrated luminosity, and employed for initial detector performance studies. They will allow basic measurements of the $B$ mesons properties; precise mass and lifetime determinations will in turn be used for inner detector calibration and alignment. Having rather clean topologies these channels constitute useful laboratories for development of data analysis procedures, such as performance calibration and optimization of flavor tagging methods. Furthermore they will serve as reference channels for rare $b$ decay searches.

Figure 5 illustrates the expected early data mass distributions for the three $B$ meson species. The exclusive cross section measurement is complementary to the inclusive determinations, involving different systematic uncertainties and model dependencies. An accuracy of the order $10 \%$ on the differential cross section $d \sigma\left(B_{u, d}\right) / d p_{T}$ and lifetime measurements is expected with about $10 \mathrm{pb}^{-1}$ [5]. The $B_{s}$ channel is of particular physics interest, allowing the extraction of the mass and width differences between the weak eigenstates, $B_{s}^{L}$ and $B_{s}^{H}$. Assuming a width difference of $20 \%$ an uncertainty on $\Delta \Gamma_{s}$ of $4 \%$ is expected for $1.3 \mathrm{fb}^{-1}$ by CMS [9]. The same channel is still the golden mode for extracting the $B_{s}$ mixing phase, which may be sizably enhanced by new physics contributions relative to the SM.

\section{$6.2 B \rightarrow \mu \mu$}

The hitherto unobserved decay $B_{s, d} \rightarrow \mu \mu$ can be a powerful tool to probe for physics beyond the SM. The channel has a tiny expected branching fraction within the SM, since it involves flavor changing neutral current transitions and is further helicity suppressed. The branching fraction may however receive substantial corrections from various of the new physics states which may be attained at the LHC. The $B_{d}$ mode having an expected branching ratio of an order of magnitude lower, focus is placed initially in the $B_{s} \rightarrow \mu \mu$ meson decay.

Whilst ATLAS and CMS do not expect to observe this decay during the early stages of the LHC, as more luminosity becomes available and the understanding of the backgrounds improves, it should be possible to identify a signal for this process. For an integrated luminosity of $10 \mathrm{fb}^{-1}$ an 

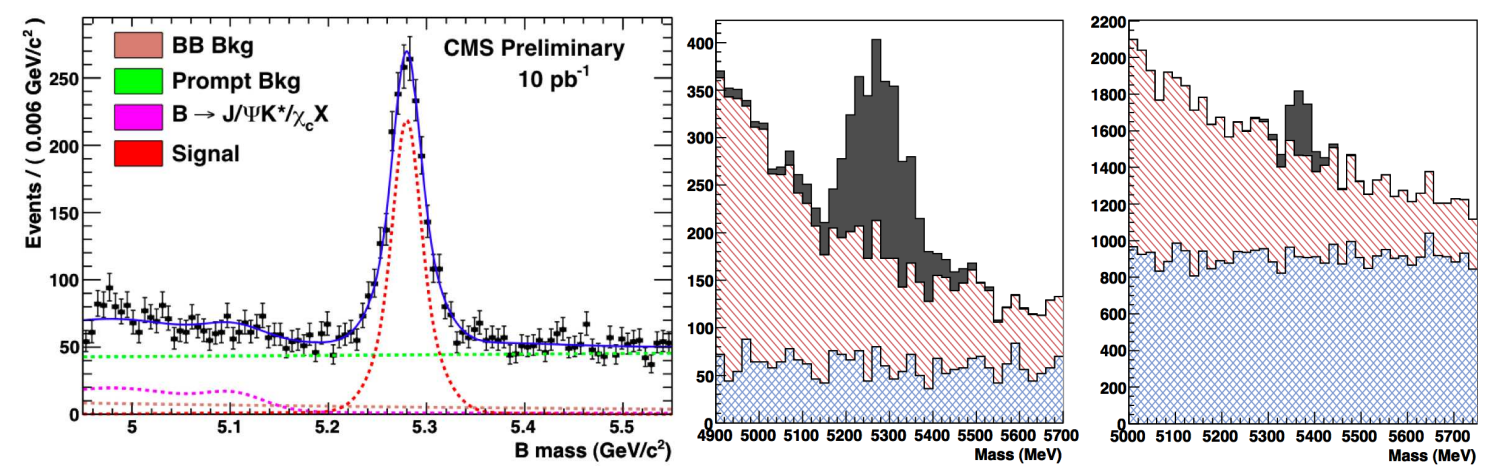

Figure 5: Expected early data $B$ meson mass distributions, reconstructed in the exclusive modes: left, $B_{u} \rightarrow$ $J / \psi K^{+} 10 \mathrm{pb}^{-1}$ (CMS); center, $B_{d} \rightarrow J / \psi K^{* 0} 10 \mathrm{pb}^{-1}$ (ALTAS); right, $B_{s} \rightarrow J / \psi \phi 150 \mathrm{pb}^{-1}$ (ATLAS).

ATLAS study [5] shows a signal expectation of 5.7 events with a background of $14_{-10}^{+13}$ events. For CMS [10] a signal of 2.4 events with a background of $6.5 \pm 2.4$ events is expected for an integrated luminosity of $1 \mathrm{fb}^{-1}$, which, by normalizing to the control $B \rightarrow J / \psi K$ channel, corresponds to an upper limit on the branching fraction for $B_{s} \rightarrow \mu \mu$ of about $1.6 \cdot 10^{-8}$ at $90 \%$ confidence level.

\section{Conclusion}

The capabilities of the two general purpose experiments ATLAS and CMS to exploit the copious heavy flavor production at the LHC at CERN are reviewed. An extensive flavor physics program has been prepared, which will contribute to the physics commissioning of the detectors with earliest collision data, and aiming in the longer run at detecting potential new physics effects in the flavor sector.

\section{References}

[1] The ATLAS Collaboration, The ATLAS experiment at the CERN LHC, JINST 3, S08003 (2008)

[2] The CMS Collaboration, The CMS experiment at the CERN LHC, JINST 3, S08004 (2008)

[3] The CDF Collaboration, Phys. Rev. Lett. 99, 132001 (2007)

[4] The D0 Collaboration, Phys. Rev. Lett. 101, 182004 (2008)

[5] The ATLAS Collaboration, Expected performance of the ATLAS experiment: detector, trigger and physics, CERN-OPEN-2008-020 (2009)

[6] The CMS Collaboration, Feasibility study of a J/ $\psi$ cross section measurement with early CMS data, CMS PAS BPH-07-002 (2008)

[7] The CMS Collaboration, Measurement of the azimuthal correlation in b $\bar{b}$ production in $p p$ collisions with the CMS detector, CMS PAS BPH-08-004 (2009)

[8] The CMS Collaboration, Measurement of open beauty production at LHC with CMS, CMS Note 2006-120 (2006)

[9] The CMS Collaboration, CMS TDR Volume II, J. Phys. G: Nuclear and Particle Physics 34, 995 (2007)

[10] The CMS Collaboration, Study of $B_{s} \rightarrow \mu^{+} \mu^{-}$in CMS, CMS PAS BPH-07-001 (2009) 